\title{
Concomitant Medication Medical History Number
}

National Cancer Institute

\section{Source}

National Cancer Institute. Concomitant Medication Medical History Number. NCI

Thesaurus. Code C83098.

The numerical identifier of the medical history event associated with the concomitant medication. 